\title{
Extraspecial Two-Groups, Generalized Yang-Baxter Equations and Braiding Quantum Gates
}

\author{
Eric C. Rowell an, Yong Zhang b:2, Yong-Shi Wu b:3, and Mo-Lin Ge c:4 \\ ${ }^{a}$ Department of Mathematics, Texas A\&M University, College Station, TX 77843 \\ ${ }^{b}$ Department of Physics and Astronomy, University of Utah, Salt Lake City, UT 84112 \\ ${ }^{c}$ Chern Institute of Mathematics, Nankai University, Tianjin 300071, China
}

\begin{abstract}
In this paper we describe connections among extraspecial 2-groups, unitary representations of the braid group and multi-qubit braiding quantum gates. We first construct new representations of extraspecial 2-groups. Extending the latter by the symmetric group, we construct new unitary braid representations, which are solutions to generalized Yang-Baxter equations and use them to realize new braiding quantum gates. These gates generate the GHZ (GreenbergerHorne-Zeilinger) states, for an arbitrary (particularly an odd) number of qubits, from the product basis. We also discuss the Yang-Baxterization of the new braid group representations, which describes unitary evolution of the GHZ states. Our study suggests that through their connection with braiding gates, extraspecial 2-groups and the GHZ states may play an important role in quantum error correction and topological quantum computing.
\end{abstract}

PACS numbers: 02.10.Kn, 03.65.Ud, 03.67.Lx

MSC 2000 numbers: 81P68 (Primary) 20F36, 20C35, 81R05 (Secondary)

Key Words: Yang-Baxter, Extraspecial 2-groups, GHZ State

\footnotetext{
${ }^{1}$ rowell@math.tamu.edu

${ }^{2}$ zhangyo2008@gmail.com. The present address: Center for High Energy Physics, Peking University, Beijing 10087, China

${ }^{3}$ wu@physics.utah.edu

${ }^{4}$ geml@nankai.edu.cn
} 


\section{Introduction}

Recently, the study of braiding quantum gates has attracted much attention [1, 2, 3, 4, 5, 6, 7, 8]. The Bell matrix generating all the Bell states from the product basis (or the computational basis) was recognized [1] to be a four-by-four solution to the braided Yang-Baxter equation (YBE) [9, 10]; later it was shown to provide a universal (two-qubit) quantum gate [2]. Higher dimensional solutions to the YBE, called the generalized Bell matrices in [8], were used to introduce the braiding quantum gates that generate the Greenberger-Horne-Zeilinger (GHZ) states [11] for an even number of qubits from the product basis. In addition, Yang-Baxterization [12, 13] of the generalized Bell matrix has been used [8] to derive a Hamiltonian that describes unitary evolution of the GHZ states of an even number of qubits.

The GHZ states are known to be the simplest multipartite maximally entanglement sources and have been widely exploited in quantum information theory (see, e.g. [14, 15, 16]). In particular, the GHZ states may act as ancillas in faulttolerant operations [17]. In this paper we study some algebraic and topological aspects of the braiding quantum gates that generate the GHZ states, for an arbitrary (particularly an odd) number of qubits. The motivation is to help establish connections between the GHZ states and topological quantum information processing and topological quantum computation, in which the braiding quantum gates are known to play a pivotal role [2, 18, 19, 20, 21]. Essentially our present paper presents generalization of a recent paper by Franko, Rowell and Wang [5]. In that paper the images of the unitary braid group representations generated by the $4 \times 4$ Bell matrix, corresponding to a two-qubit braiding quantum gate [2], have been identified through extension of representations of the extraspecial 2 -groups. The decomposition of reducible braid group representations into their irreducible constituents have also been determined, and it has been shown to be closely related to the well-known Jones representations [22, 23] at 4th roots of unity, associated with the $S U(2)_{2}$ model for topological matter states (see, e.g. [24]). In the present paper we generalize these results to the construction - again through extraspecial 2-groups - of higher dimensional unitary braid group representations that give rise to the braiding quantum gates acting on an arbitrary (particularly an odd) number of qubits and generating multi-qubit GHZ states from the product basis. We also carry out the Yang-Baxterization of the new braid group representations, which can be used to describe unitary evolution of the GHZ states of any number of qubits. Possible connections with quantum error correction and topological quantum computation will be discussed in the concluding section.

This paper is organized as follows. In Section 2 we record our notational conventions and introduce the relevant braid groups and a generalized form of the Yang-Baxter Equation. Section 3 contains the main mathematical results describing how one obtains unitary representations of braid groups from those of extraspecial 2-groups and how the latter may be constructed from almost- 
complex structures. In Section 4 we explain how to obtain GHZ states from the product (computational) basis using the braid representations found in Section 3 , as well as their unitary evolution under the Hamiltonians derived via YangBaxterization [12, 13]. Section 5 is devoted to concluding remarks on relevance of extraspecial 2-groups and the GHZ states to quantum error correction and topological quantum computation, while the Appendix describes for interested readers a generalized version of the quantum Yang-Baxter equation developed in this paper.

\section{Preliminaries}

We use $\mathbb{1}$ to denote the identity operator and $\mathbb{1}_{m}$ the $m \times m$ identity matrix. The superscripts $*$ and $\dagger$, respectively, denote the complex conjugation and Hermitian operation of a matrix (or a complex number). The symbol $\delta_{i j}$ is the Kronecker function of two variables $i, j$, which is 1 if $i=j$ and 0 otherwise. The function $\epsilon(k)$ is defined by

$$
\epsilon(k) \epsilon(k)=1, \quad \epsilon(k) \epsilon(\bar{k})=-1,
$$

which has solutions $\epsilon(k)= \pm 1, \epsilon(\bar{k})=\mp 1$, and either $\bar{k}=-k$ or $\bar{k}=2 n-k+1$ depending on the convention used for the range of $k$ or $\bar{k}$. The tensor product $A \otimes B$ of the matrices $A=\left(A_{i j}\right)$ and $B=\left(B_{k l}\right)$ is defined by the convention $(A \otimes B)_{i k, j l}=A_{i j} B_{k l}$.

The symbol $A^{J_{1} J_{2}}$ denotes a matrix having the following matrix entries,

$$
\left(A^{J_{1} J_{2}}\right)_{\mu a, \nu b}, \mu, \nu=J_{1}, J_{1}-1, \cdots,-J_{1}, a, b=J_{2}, J_{2}-1, \cdots,-J_{2},
$$

where $J_{1}, J_{2}$ are integers or half-integers. The matrix $A^{J_{1} J_{2}}$ has the following operator presentation, also denoted as $A^{J_{1} J_{2}}$, in terms of Dirac kets and bras:

$$
A^{J_{1} J_{2}}=\sum_{\mu=-J_{1}}^{J_{1}} \sum_{\nu=-J_{1}}^{J_{1}} \sum_{a=-J_{2}}^{J_{2}} \sum_{b=-J_{2}}^{J_{2}}\left(A^{J_{1} J_{2}}\right)_{\mu a, \nu b}|\mu a\rangle\langle\nu b|,
$$

where the kets $\mid\{\mu\rangle\}$ or $\{|a\rangle\}$ form an orthonormal basis, respectively, in $2 J_{1}+1$ and $2 J_{2}+1$ dimensional Hilbert space:

$$
\sum_{\mu=-J_{1}}^{J_{1}}|\mu\rangle\left\langle\mu\left|=\mathbb{1}_{\left(2 J_{1}+1\right)}, \quad \sum_{a=-J_{2}}^{J_{2}}\right| a\right\rangle\langle a|=\mathbb{1}_{\left(2 J_{2}+1\right)},
$$

where $\langle\mu \mid \nu\rangle=\delta_{\mu \nu}$ and $\langle a \mid b\rangle=\delta_{a b}$.

The Pauli matrices $\sigma_{x}, \sigma_{y}, \sigma_{z}$ have the conventional forms

$$
\sigma_{x}=\left(\begin{array}{cc}
0 & 1 \\
1 & 0
\end{array}\right), \quad \sqrt{-1} \sigma_{y}=\left(\begin{array}{cc}
0 & 1 \\
-1 & 0
\end{array}\right), \quad \sigma_{z}=\left(\begin{array}{cc}
1 & 0 \\
0 & -1
\end{array}\right) .
$$


Artin's braid group $\mathcal{B}_{n}$ on $n$ strands has the well-known presentation in terms of generators $b_{1}, \ldots, b_{n-1}$ satisfying the far-commutation relation

$$
b_{i} b_{j}=b_{j} b_{i}, \quad|i-j| \geq 2
$$

and the braid relation

$$
b_{i} b_{i+1} b_{i}=b_{i+1} b_{i} b_{i+1}, \quad 1 \leq i \leq n-2 .
$$

$\mathcal{B}_{n}$ has a finite-index normal subgroup $\mathcal{P}_{n}$ generated by the conjugacy class of $\left(b_{1}\right)^{2} . \mathcal{P}_{n}$ is called the pure braid group and can be understood as the kernel of the surjective homomorphism $\mathcal{B}_{n} \rightarrow \mathcal{S}_{n}$ onto the symmetric group on $n$ letters sending the generators $b_{i}$ of $\mathcal{B}_{n}$ to transpositions $(i i+1)$. This induces an isomorphism $\mathcal{S}_{n} \cong \mathcal{B}_{n} / \mathcal{P}_{n}$. The term "pure braids" applied to elements of $\mathcal{P}_{n}$ is due to the fact that in the geometric formulation of $\mathcal{B}_{n}$ as braiding operators on $n$ strands, the elements of $\mathcal{P}_{n}$ are exactly those that do not permute the strands.

Relation (2.7) leads to the (braided version of the) YBE, i.e.,

$$
\left(\check{R} \otimes \mathbb{1}_{d}\right)\left(\mathbb{1}_{d} \otimes \check{R}\right)\left(\check{R} \otimes \mathbb{1}_{d}\right)=\left(\mathbb{1}_{d} \otimes \check{R}\right)\left(\check{R} \otimes \mathbb{1}_{d}\right)\left(\mathbb{1}_{d} \otimes \check{R}\right),
$$

with an invertible $d^{2} \otimes d^{2}$ matrix $\check{R}: V \otimes V \rightarrow V \otimes V$ where $V=\mathbb{C}^{d}$. The relation (2.8) gives rise to a sequence of representations $\left(\pi_{n},\left(\mathbb{C}^{d}\right)^{\otimes n}\right)$ of $\mathcal{B}_{n}$ :

$$
\pi_{n}\left(b_{i}\right)=\mathbb{1}_{d}^{\otimes i-1} \otimes \check{R} \otimes \mathbb{1}_{d}^{\otimes n-i-1}
$$

since clearly $\pi_{n}\left(b_{i}\right)$ and $\pi_{n}\left(b_{j}\right)$ commute for $|i-j| \geq 2$. This type of braid group representations was used in [8] to construct the GHZ states for an even number of qubits.

To construct the GHZ for an odd number of qubits, we will explore solutions to a generalized version of the YBE:

Definition 2.10. Fix $p$ with $2 \leq p \in \mathbb{N}$ and let $l=p^{k}$. An invertible $p^{N} \times p^{N}$ matrix $\check{R}$ is a solution to the generalized Yang-Baxter Equation if

$$
\left(\check{R} \otimes \mathbb{1}_{l}\right)\left(\mathbb{1}_{l} \otimes \check{R}\right)\left(\check{R} \otimes \mathbb{1}_{l}\right)=\left(\mathbb{1}_{l} \otimes \check{R}\right)\left(\check{R} \otimes \mathbb{1}_{l}\right)\left(\mathbb{1}_{l} \otimes \check{R}\right),
$$

as operators on $\left(\mathbb{C}^{p}\right)^{\otimes(k+N)}$.

When $k=1, N=2$, the generalized YBE (2.11) is the same as the conventional YBE (2.8). If $k \geq N / 2$, the assignment

$$
\pi_{n}\left(b_{i}\right)=\mathbb{1}_{l}^{\otimes i-1} \otimes \check{R} \otimes \mathbb{1}_{l}^{\otimes n-i-1}
$$

defines a sequence of representations $\left(\pi_{n},\left(\mathbb{C}^{p}\right)^{\otimes(N+k(n-2))}\right)$ of the braid group $\mathcal{B}_{n}$. Here relation (2.7) is clearly satisfied by the $\pi_{n}\left(b_{i}\right)$ but relation (2.6) necessitates the restriction $k \geq N / 2$ as we will see later. In the following we are going to consider only the case with $p=2$, corresponding to qubits.

Finally we will use the term almost-complex structure to mean a matrix $M$ such that $M^{2}=-11$. 


\section{Extraspecial 2-groups and unitary braid group rep- resentations}

In [5, extraspecial 2-groups are seen to play a key role in studying the images of the braid group $\mathcal{B}_{n}$ under the representation associated with the $4 \times 4$ Bell matrix. Inspired by this work, we present an approach to the GHZ states (higher dimensional generalizations of the Bell states) starting from extraspecial 2-groups and their anti-Hermitian representations in terms of almost-complex structures.

\subsection{Extraspecial 2-groups}

We give a brief sketch of extraspecial 2-groups following [5]. The group $\mathbf{E}_{m}$ is the abstract group generated by $e_{1}, \ldots, e_{m}$ with relations:

$$
e_{i}^{2}=-\mathbb{1}, e_{i} e_{j}=e_{j} e_{i},|i-j| \geq 2, \quad e_{i+1} e_{i}=-e_{i} e_{i+1}, 1 \leq i, j \leq m-1,
$$

Here $-\mathbb{1}$ is an order 2 central element, and we denote $-\mathbb{1} a$ by $-a$. Any element in $\mathbf{E}_{m}$ can be expressed in a unique normal form: $\pm e_{1}^{\alpha_{1}} \cdots e_{m}^{\alpha_{m}}$ where $\alpha_{i} \in \mathbb{Z}_{2}$, and $\mathbf{E}_{m} /\{ \pm \mathbb{1}\} \cong\left(\mathbb{Z}_{2}\right)^{m}$. It is clear from this normal form that $\mathbf{E}_{m}$ has order $2^{m+1}$.

A group $G$ of order $2^{m+1}$ with $m$ even is an extraspecial 2-group if both the center $Z(G)(=\{g \in G: g a=a g$ forall $a \in G\})$ and the commutator subgroup $G^{\prime}$ (generated by elements of the form $a b a^{-1} b^{-1}$ ) are isomorphic to $\mathbb{Z}_{2}$ and $G / Z(G) \cong\left(\mathbb{Z}_{2}\right)^{m}$ (see [25]). The commutator subgroup of $\mathbf{E}_{m}$ is $\{ \pm \mathbb{1}\}$ due to its (anti-)commutation relations, and hence it is immediate that $\mathbf{E}_{2 k}$ is an extraspecial 2-group. When $m$ is odd the center of $\mathbf{E}_{m}$ has order 4. However, since $\mathbf{E}_{m-1} \subset \mathbf{E}_{m} \subset \mathbf{E}_{m+1}$ we obtain an extraspecial 2-group from $\mathbf{E}_{m}$ by adding or removing a generator, so we will call $\mathbf{E}_{m}$ with $m$ odd a nearly extraspecial 2-group.

The center of $\mathbf{E}_{m}$ is

$$
Z\left(\mathbf{E}_{m}\right)=\left\{\begin{array}{cc}
\{ \pm \mathbb{1}\} & m \text { even } \\
\left\{ \pm \mathbb{1}, \pm e_{1} e_{3} \cdots e_{m}\right\} & m \text { odd }
\end{array}\right.
$$

For $m=2 k-1$ odd, the form of the center of $\mathbf{E}_{2 k-1}$ depends on the parity of $k$ :

$$
Z\left(\mathbf{E}_{2 k-1}\right) \cong\left\{\begin{array}{cc}
\mathbb{Z}_{2} \times \mathbb{Z}_{2} & k \text { even } \\
\mathbb{Z}_{4} & k \text { odd }
\end{array}\right.
$$

\subsection{Irreducible representations of $\mathbf{E}_{m}$}

Irreducible representations of $\mathbf{E}_{m}$ are described in detail in [5]. We summarize them here to establish notation, for explicit realizations, see [5]. For $m=2 k$, there are a unique irreducible $2^{k}$-dimensional representation which we denote by $\left(\rho_{1}, V_{1}\right)$, and $2^{2 k}$ inequivalent 1-dimensional representations $\left(\rho_{j}, V_{j}\right), j=$ $2, \cdots, 2^{2 k}+1$ of the form

$$
\rho_{j}( \pm \mathbb{1})=1, \quad \rho_{j}\left( \pm e_{i}\right)= \pm 1, i=1, \cdots 2 k .
$$


Note that in the right hand side of the second equation, for a fixed $i$ there are two choices of the sign; altogether there are $2^{2 k}$ choices, corresponding to $2^{2 k}$ inequivalent representations labelled by $j=2, \cdots, 2^{2 k}+1$.

For $m=2 k-1, \mathbf{E}_{m}$ has $2^{2 k-1}$ inequivalent 1-dimensional representations denoted by $\left(\lambda_{j}, W_{j}\right), j=3, \cdots, 2^{2 k-1}+2$ of the form

$$
\lambda_{j}( \pm \mathbb{1})=1, \quad \lambda_{j}\left( \pm e_{i}\right)= \pm 1, i=1, \cdots 2 k-1 .
$$

A note similar to that below eq. (3.4) applies here. Moreover, $\mathbf{E}_{2 k-1}$ has two inequivalent irreducible $2^{k-1}$-dimensional representations $\left(\lambda_{1}, W_{1}\right)$ and $\left(\lambda_{2}, W_{2}\right)$.

The following key proposition shows that certain representations of $\mathbf{E}_{n-1}$ induce representations of $\mathcal{B}_{n}$ :

Proposition 3.6. Let $\left\{T_{1}, \ldots, T_{n-1}\right\}$ be a set of $k \times k$ matrices such that:

(E1) $T_{i}^{2}=-\mathbb{1}$,

(E2) $T_{i} T_{j}=T_{j} T_{i}$ if $|i-j|>1$,

(E3) $T_{i} T_{i+1}=-T_{i+1} T_{i}$ for all $1 \leq i \leq(n-2)$.

Then

(a) The assignment $\phi_{n-1}\left(e_{i}\right)=T_{i}$ defines a representation of $\mathbf{E}_{n-1}$. Moreover, $\phi_{n-1}$ contains no 1-dimensional subrepresentations.

(b) Let $\check{R}_{i}=\frac{1}{\sqrt{2}}\left(\mathbb{1}+T_{i}\right), 1 \leq i \leq n-1$. Then $b_{i} \rightarrow \check{R}_{i}$ defines a representation of $\mathcal{B}_{n}$.

(c) If in addition the $T_{i}$ are anti-Hermitian (i.e. $T_{i}=-T_{i}^{\dagger}$ ), the $\mathcal{B}_{n}$ representation is unitary.

(d) Moreover, if $n$ is odd, $\phi_{n-1}$ is a direct sum of $\frac{\operatorname{dim}\left(\phi_{n-1}\right)}{2^{(n-1) / 2}}$ copies of $\rho_{1}$.

Proof. Since the $T_{i}$ satisfy (3.1) it is clear that $\phi_{n-1}$ does define a representation of $\mathbf{E}_{n-1}$. If the central element $-\mathbb{1} \in \mathbf{E}_{n-1}$ has image $-\mathbb{1}$ then this holds for any subrepresentation of $\phi_{n-1}$ as well. By the explicit construction of 1-dimensional representations of $\mathbf{E}_{n-1}$ in [5], $\phi_{n-1}$ has no 1-dimensional representations since $-\mathbb{1}$ acts by 1 for these representations. This proves (a). Claim (d) follows immediately from (a), since for $n-1$ even there is only one irreducible representation of dimension more that 1 , and its dimension is $2^{(n-1) / 2}$.

The matrices $\check{R}_{i}$ obviously satisfy relation (2.6) since the $T_{i}$ satisfy (E2). Moreover, we have:

$$
\begin{aligned}
\sqrt{2} \check{R}_{i} \check{R}_{i+1} \check{R}_{i} & =T_{i} T_{i+1} T_{i}+2 T_{i}+T_{i+1}+T_{i} T_{i+1}+T_{i+1} T_{i}+T_{i}^{2}+\mathbb{1} \\
& =2 T_{i+1}+2 T_{i}+T_{i} T_{i+1}+T_{i+1} T_{i}=2 T_{i+1}+2 T_{i},
\end{aligned}
$$

which is symmetric under $i \leftrightarrow i+1$ so that the $\check{R}_{i}$ satisfy the braid relation (2.7), proving (b).

Observing that $\check{R}_{i}^{-1}=\frac{1}{\sqrt{2}}\left(\mathbb{1}-T_{i}\right)$ it is clear that if the $T_{i}$ are anti-Hermitian then the $\check{R}_{i}$ are unitary, giving (c). 
Remark 3.7. Note that if the $T_{i}$ satisfy $(E 1)$ and $(E 2)$ then the matrices $\check{R}_{i}=$ $\frac{1}{\sqrt{2}}\left(\mathbb{1}+T_{i}\right)$ satisfy (2.6) automatically, and satisfy (2.7) precisely when

$$
\left(E 3^{\prime}\right) \quad T_{i}+T_{i} T_{i+1} T_{i}-T_{i+1}-T_{i+1} T_{i} T_{i+1}=0 .
$$

While $(E 3)$ is sufficient to imply $\left(E 3^{\prime}\right)$, one wonders if there are other interesting group relations or almost-complex structures that satisfy $\left(E 3^{\prime}\right)$ but not $(E 3)$.

Let $\phi_{n-1}$ be any representation of $\mathbf{E}_{n-1}$ satisfying the hypotheses of Proposition 3.6 and such that $\phi_{n-1}\left(e_{i}\right)$ is anti-Hermitian. Then we obtain unitary representations $\pi_{n}$ of the braid group $\mathcal{B}_{n}$ via:

$$
\pi_{n}\left(b_{i}\right)=\frac{1}{\sqrt{2}}\left(\mathbb{1}+\phi_{n-1}\left(e_{i}\right)\right) .
$$

We wish to describe the images of $\pi_{n}$ as in [5], which is in fact a special case of our analysis. Note the following key relations between $\pi_{n}\left(\mathcal{B}_{n}\right)$ and $\phi_{n}\left(\mathbf{E}_{n-1}\right)$ :

$$
\begin{aligned}
& \pi_{n}\left(b_{i}\right) \phi_{n-1}\left(e_{i \pm 1}\right) \pi_{n}\left(b_{i}\right)^{-1}=\phi_{n-1}\left(e_{i}\right) \phi_{n-1}\left(e_{i \pm 1}\right), \\
& \pi_{n}\left(b_{i}\right) \phi_{n-1}\left(e_{j}\right) \pi_{n}\left(b_{i}\right)^{-1}=\phi_{n-1}\left(e_{j}\right)
\end{aligned}
$$

where $1 \leq i \pm 1 \leq n-1$ and $|i-j| \geq 2$. Observing that $\left[\pi_{n}\left(b_{i}\right)\right]^{2}=\phi_{n-1}\left(e_{i}\right)$ these relations immediately imply that the restriction of $\pi_{n}$ to the normal subgroup $\mathcal{P}_{n}$ is $H_{n}:=\pi_{n}\left(\mathcal{P}_{n}\right)=\phi_{n-1}\left(\mathbf{E}_{n-1}\right)$ since $\mathcal{P}_{n}$ is generated by all conjugates of $\left(b_{i}\right)^{2}$.

Now let us show that the braid group image $G_{n}:=\pi_{n}\left(\mathcal{B}_{n}\right)$ is an extension of $\mathbf{E}_{n-1}$ by the symmetric group $\mathcal{S}_{n}$. We must show that the surjective homomorphism $\pi_{n}^{\prime}: \mathcal{S}_{n} \rightarrow G_{n} / H_{n}$ is in fact a bijection. For this consider the homomorphism $\vartheta: G_{n} \rightarrow \operatorname{Aut}\left(H_{n}\right)$ from $G_{n}$ to the automorphism group of $H_{n}$ where $\vartheta\left(G_{n}\right)$ acts by conjugation (explicitly by (3.9) $)$. Note that since $\vartheta\left(H_{n}\right)=: \operatorname{Inn}\left(H_{n}\right)$, the subgroup of inner automorphisms, the map $\vartheta$ induces a homomorphism $\bar{\vartheta}: G_{n} / H_{n} \rightarrow \operatorname{Out}\left(H_{n}\right):=\operatorname{Aut}\left(H_{n}\right) / \operatorname{Inn}\left(H_{n}\right)$. Clearly $\operatorname{Inn}\left(H_{n}\right)$ acts on $H_{n}$ by sign changes. To show that $\pi_{n}^{\prime}$ is injective, it is enough to show that $\bar{\vartheta} \circ \pi_{n}^{\prime}: \mathcal{S}_{n} \rightarrow \operatorname{Out}\left(H_{n}\right)$ is injective, i.e. $\operatorname{ker}\left(\bar{\vartheta} \circ \pi_{n}^{\prime}\right) \triangleleft S_{n}$ is trivial. Since the only nontrivial proper normal subgroup of $\mathcal{S}_{n}$ is $A_{n}$ for $n \geq 5$, it is enough to show that the images of the permutations (12), (123) and (12)(34) (for $n=4)$ under $\bar{\vartheta}$ are nontrivial-easily accomplished using (3.9). Thus we have an exact sequence:

$$
\mathbb{1} \rightarrow H_{n} \rightarrow G_{n} \rightarrow \mathcal{S}_{n} \rightarrow \mathbb{1},
$$

for all $n \geq 2$, where $H_{n} \rightarrow G_{n}$ is inclusion and $G_{n} \rightarrow \mathcal{S}_{n}$ is induced by the quotient map and the isomorphism $\pi_{n}^{\prime}$.

\subsection{Representations of $\mathrm{E}_{m}$ via almost-complex structures}

Now we introduce almost-complex structures and construct representations of nearly extraspecial 2-groups satisfying the hypotheses of Proposition 3.6, which will then give rise to representations of $\mathcal{B}_{n}$ generalizing those of [5]. 
First we consider a $2 n \times 2 n$ anti-Hermitian matrix $M_{2 n}$ of the form

$$
M_{2 n}=\sum_{i=1}^{2 n} \epsilon(i)|i\rangle\langle\bar{i}|, \quad \bar{i}=2 n+1-i,
$$

where the function $\epsilon(i)$ satisfy Eqs. (2.1), and the Dirac kets $|i\rangle$ form an orthonormal basis. $M_{2 n}$ satisfies $\left(M_{2 n}\right)^{2}=-\mathbb{1}_{2 n}$ and $\left(M_{2 n}\right)^{\dagger}=-M_{2 n}$.

In what follows, we construct two classes of representations $\phi_{m}^{(i)}, i=1,2$ for the group $\mathbf{E}_{m}$ in terms of the almost-complex structure $M_{2 n}$. The first class has already appeared in [8], but we summarize it here for comparison.

Class (1): The almost-complex structure to be used is a $(2 k)^{2} \times(2 k)^{2}$ matrix $M^{J J}$ with complex deformation parameters $q_{i j} \in \mathbb{C}$,

$$
M^{J J}=\sum_{i, j=-J}^{J} \epsilon(i) q_{i j}|i j\rangle\langle\bar{i} \bar{j}|, \quad \bar{i}=-i, \bar{j}=-j, \quad J=k-\frac{1}{2}, k \in \mathbb{N}
$$

where the $\epsilon(i)$ may be arbitrarily chosen subject to (2.1). These matrices have already appeared in [8] for describing the GHZ states of an even number of objects. For completeness we include the following, which is implicit in [8]:

Theorem 3.13. Define a map $\phi_{m}^{(1)}$ on the generators of $\mathbf{E}_{m}$ in terms of $M^{J J}$ by:

$$
\phi_{m}^{(1)}\left(e_{i}\right)=\mathbb{1}_{2 k}^{\otimes i-1} \otimes M^{J J} \otimes \mathbb{1}_{2 k}^{\otimes m-i}, \quad i=1, \cdots, m .
$$

Then $\phi_{m}^{(1)}$ defines a $(2 k)^{m+1}$-dimensional unitary representation of $\mathbf{E}_{m}$ if and only if the parameters $q_{i j}$ in $M^{J J}$ satisfy the following three constraints:

$$
q_{i j} q_{\bar{i} \bar{j}}=1, \quad q_{i j} q_{\bar{i} j}=q_{j l} q_{j \bar{l}}, \quad q_{i j}^{*} q_{i j}=1 .
$$

Proof. One checks that $T_{i}:=\phi_{m}^{(1)}\left(e_{i}\right)$ is anti-Hermitian and satisfies (E1) and (E3) of Proposition 3.6 if and only if relations (3.15) hold. Relation (E2) is immediate.

In the spirit of separation of variables, we assume $q_{i j}=q_{i} q_{j}$ and $q_{i} \neq 0$ to obtain solutions of (3.15),

$$
q_{i} q_{\bar{i}}=1, \quad q_{i}^{*}=q_{\bar{i}}, \quad q_{i} \in \mathbb{C}
$$

and recast $M^{J J}$ into a tensor product of two matrices $M_{2 k}^{\prime}$ and $P_{2 k}^{\prime}$,

$$
M^{J J}=M_{2 k}^{\prime} \otimes P_{2 k}^{\prime}, \quad M_{2 k}^{\prime}=\sum_{i=-J}^{J} \epsilon(i) q_{i}|i\rangle\left\langle\bar{i}\left|, P_{2 k}^{\prime}=\sum_{j=-J}^{J} q_{j}\right| j\right\rangle\langle\bar{j}| .
$$

These matrices then satisfy $\left(M_{2 k}^{\prime}\right)^{2}=-\mathbb{1}_{2 k}$ and $\left(M_{2 k}^{\prime}\right)^{\dagger}=-M_{2 k}^{\prime}$ as well as

$$
\left(P_{2 k}^{\prime}\right)^{2}=\mathbb{1}_{2 k}, \quad\left(P_{2 k}^{\prime}\right)^{\dagger}=P_{2 k}^{\prime}, \quad M_{2 k}^{\prime} P_{2 k}^{\prime}=-P_{2 k}^{\prime} M_{2 k}^{\prime} .
$$


Remark 3.19. It should be pointed out that from an algebraic point of view we may assume:

1. $q_{i}=1$ for all $i$ and

2. $\epsilon(i)=1$ for $i>0$ and $\epsilon(i)=-1$ for $i<0$.

The reason is that for any choice of $q_{i}$ and any admissible choice of signs $\epsilon(i)$ the representations of $\mathbf{E}_{m}$ defined via $M^{J J}$ is unitarily equivalent to those representations of $\mathbf{E}_{m}$ obtained from any other choice of $q_{i}$ and $\epsilon(i)$. That is, we may find a global unitary change of basis (via a diagonal matrix in fact) with respect to which the matrices $M_{2 k}^{\prime}$ and $P_{2 k}^{\prime}$ may be assumed to have the form:

$$
M_{2 k}=\sum_{i=-J}^{J} \epsilon(i)\left|i^{\prime}\right\rangle\left\langle\bar{i}^{\prime}\left|, \quad P_{2 k}=\sum_{i=-J}^{J}\right| i^{\prime}\right\rangle\left\langle\bar{i}^{\prime}\right|
$$

where $\epsilon(i)$ is as above.

On the other hand, these unimodular deformation parameters can be understood as phase factors which play key roles in quantum mechanics, for example, the angle variable $\varphi$ at the deformation parameter $q_{\frac{1}{2} \frac{1}{2}}$ in $M^{\frac{1}{2} \frac{1}{2}}$ is explained as an angle parameter for the rotation in the Bloch sphere, see [3, 4]. Moreover, these unimodular deformation parameters have an interpretation in terms of Berry phases in quantum mechanics [26]. The connection to the Berry phase is the following: Such phases usually can be removed by suitable unitary transformations and hence are thought of as spurious; but under certain conditions in an adiabatic evolution they give rise to non-trivial boundary effects after periodic closure. (For the possible relations to boundary issues (in the context of braiding gates), see also refs. [27, 28, 29].)

Class (2): There are two natural ways in which to generalize Class (1) in search of representations of $\mathbf{E}_{m}$. Firstly, we may consider more general almostcomplex structures of the form $M_{2 k_{1}} \otimes P_{2 k_{2}}$ where $k_{1} \neq k_{2}$. Secondly, we look for solutions to the generalized YBE that satisfy both (2.6) and (2.7). Taken in tandem, this is a formidable problem. For simplicity and with an eye towards GHZ states, we consider a special case: $k_{1}=1$ and $k_{2}=2^{N-2}, N \geq 2$. In particular we define

$$
M_{2^{N}}=M_{2} \otimes P_{2^{N-1}}=\sqrt{-1} \sigma_{y} \otimes \sigma_{x}^{\otimes N-1} .
$$

Notice that this $M_{2^{N}}$ does not depend on any deformation parameters or sign choices $\epsilon(i)$. Having fixed this $M_{2^{N}}$ we can construct another class of representations of $\mathbf{E}_{m}$, as summarized by the following main result:

Theorem 3.21. Define $\phi_{m}^{(2)}$ on generators of $\mathbf{E}_{m}$ by

$$
\phi_{m}^{(2)}\left(e_{i}\right)=\mathbb{1}_{2^{k}}^{\otimes i-1} \otimes \sqrt{-1} \sigma_{y} \otimes \sigma_{x}^{\otimes N-1} \otimes \mathbb{1}_{2^{k}}^{\otimes m-i}, \quad i=1, \cdots, m .
$$

Then $\phi_{m}^{(2)}$ defines an (anti-Hermitian) representation of $\mathbf{E}_{m}$ into $\mathrm{U}\left(2^{N+k(m-1)}\right)$ for all $m \geq 2$ if and only if $\frac{N}{2} \leq k \leq N-1$. 
Proof. One easily checks that $M_{2^{N}}=\sqrt{-1} \sigma_{y} \otimes \sigma_{x}^{\otimes N-1}$ is anti-Hermitian and satisfied $M_{2^{N}}^{2}=-\mathbb{1}_{2^{N}}$. This implies that the $\phi_{m}^{(2)}\left(e_{i}\right)$ are also anti-Hermitian and satisfy

$$
\left[\phi_{m}^{(2)}\left(e_{i}\right)\right]^{2}=-\mathbb{1}_{2^{N+k(m-1)}} .
$$

Direct calculation shows that $\phi_{m}^{(2)}\left(e_{i}\right)$ and $\phi_{m}^{(2)}\left(e_{j}\right)$ with $i \leq j$ anti-commute if and only if $1 \leq k(j-i) \leq N-1$, and commute if and only if $N-1<k(j-i)$. Taking $j=i+1$ in the first condition yields $1 \leq k \leq N-1$, while for $m \geq 3$ taking $j=i+2$ in the second condition produces $\frac{N}{2} \leq k \leq N-1$.

We remark that while $\phi_{m}^{(2)}$ is a 2-parameter family of representations depending on $N$ and $k$, we have suppressed this dependence for notational convenience.

\subsection{Decomposition into irreducible representations of $\mathcal{B}_{n}$}

We proceed to determine the decomposition of the unitary braid group representations obtained from the representations $\phi_{n-1}^{(i)}(i=1,2)$ of $\mathbf{E}_{n-1}$ into irreducible constituents. Since these $\phi^{(i)}$ satisfy the hypotheses of Proposition 3.6, we have the conclusions at our disposal. Moreover, the classification and formulas for irreducible representations $\rho_{1}, \lambda_{1}$ and $\lambda_{2}$ of group $\mathbf{E}_{n-1}$ are given in [5], so one can easily compute their (irreducible, since the restrictions to $\mathcal{P}_{n}$ are) extensions $\hat{\rho}_{1}, \hat{\lambda}_{1}$ and $\hat{\lambda}_{2}$ to $\mathcal{B}_{n}$.

We consider Class (1) and Class (2) simultaneously, so that the representation $\pi_{n}$ is induced from $\phi_{n-1}^{(i)}$ with $i=1$ or $i=2$. First let us consider the decomposition of $\pi_{n}$ of $\mathcal{B}_{n}$ with $n$ odd, so that the restriction of $\pi_{n}$ to $\mathcal{P}_{n}$ factors over the representation $\phi_{n-1}^{(i)}$ of $\mathbf{E}_{n-1}$ with $n-1$ even. Set $d_{i}^{o}=\frac{\operatorname{dim}\left(\phi_{n-1}^{(i)}\right)}{2^{(n-1) / 2}}$. Then Proposition 3.6 (d) implies that $\phi_{n-1}^{(i)}$ decomposes into irreducible subrepresentations as $d_{i}^{o}$ copies of the $2^{(n-1) / 2}$-dimensional irreducible $\mathbf{E}_{n-1}$-representation $\left(\rho_{1}, V_{1}\right)$. Thus $\pi_{n}$ decomposes as $d_{i}^{o}$ copies of $\hat{\rho}_{1}$ as representations of $\mathcal{B}_{n}$. Now consider $n$ even, and set $d_{i}^{e}=\frac{\operatorname{dim}\left(\phi_{n-1}^{(i)}\right)}{2^{n / 2}}$. It was observed in [5] that the restriction of $\rho_{1}$ to $\mathbf{E}_{n-1}$ decomposes as follows:

$$
\operatorname{Res}_{\mathbf{E}_{n-1}}^{\mathbf{E}_{n}}\left(\rho_{1}, V_{1}\right)=\left(\lambda_{1}, W_{1}\right) \oplus\left(\lambda_{2}, W_{2}\right) .
$$

This together with Proposition 3.6(a) shows that $\phi_{n-1}^{(i)}$ decomposes as a representation of $\mathbf{E}_{n-1}$ into $d_{i}^{e}$ copies of $\lambda_{1} \oplus \lambda_{2}$ where $\lambda_{i}, i=1,2$ are the two inequivalent irreducible representations of $\mathbf{E}_{n-1}$ with $n$ even with $\operatorname{dim}\left(\lambda_{i}\right)=2^{(n-2) / 2}$. Thus the $\mathcal{B}_{n}$ representation $\pi_{n}$ with $n$ even decomposes as $d_{i}^{e}$ copies of $\hat{\lambda}_{1} \oplus \hat{\lambda}_{2}$. For completeness, let us recall that $\operatorname{dim}\left(\phi_{n-1}^{(1)}\right)=(2 k)^{n}$ and $\operatorname{dim}\left(\phi_{n-1}^{(2)}\right)=2^{N+k(n-2)}$ so that $d_{1}^{o}=k^{n} 2^{(n+1) / 2}$ and $d_{2}^{o}=2^{N+k(n-2)-(n-1) / 2}$ with $d_{i}^{e}$ computed similarly.

In [5] it was shown that a renormalization of the $4 \times 4$ Bell basis-change matrix leads to a (projectively equivalent) representation of $\mathcal{B}_{n}$ that factors over the wellknown Jones representation at a 4th root of unity (via Temperley-Lieb algebras). 
It is clear that the generalized Bell matrices $B_{2 k}$ (with the case in [5] as a special case) may be renormalized in the same fashion to obtain the same conclusion. In particular, the matrices $B_{2 k}$ may be used to define link-invariants, which will contain the same topological information as the Jones polynomial [22, 23] at a 4 th root of unity.

\section{GHZ states and their unitary evolution via QYBE}

In [8], it was observed that GHZ states (corresponding to an even number of qubits) can be obtained from the product basis via operators that satisfy the (conventional) YBE (2.8). In what follows we put the results of [8] into the present context and describe the role played by the generalized YBE (2.11) in producing GHZ states with an odd number of qubits. Moreover, Yang-Baxterization [12, 13. is exploited to obtain specific Hamiltonians that give rise to unitary evolution of the GHZ states.

\subsection{Unitary basis transformation matrices}

The two dimensional Hilbert space $\mathcal{H}_{2}$ spanned by eigenvectors $|m\rangle, m= \pm \frac{1}{2}$ of the spin- $\frac{1}{2}$ operators (i.e. Pauli matrices, for example, $\sigma_{z}\left| \pm \frac{1}{2}\right\rangle= \pm\left| \pm \frac{1}{2}\right\rangle$ ), has the following realization of coordinate vectors over the complex field $\mathbb{C}^{2}$,

$$
\left|\frac{1}{2}\right\rangle:=\left(\begin{array}{l}
1 \\
0
\end{array}\right), \quad\left|-\frac{1}{2}\right\rangle:=\left(\begin{array}{c}
0 \\
1
\end{array}\right), \quad \alpha\left|\frac{1}{2}\right\rangle+\beta\left|-\frac{1}{2}\right\rangle=\left(\begin{array}{c}
\alpha \\
\beta
\end{array}\right),
$$

which determine actions of $\sqrt{-1} \sigma_{y}$ and $\sigma_{x}$ on the basis $|m\rangle$,

$$
\sigma_{x}|m\rangle=|\bar{m}\rangle, \quad \sqrt{-1} \sigma_{y}|m\rangle=\epsilon^{\prime}(m)|\bar{m}\rangle, \quad \bar{m}=-m, \quad m= \pm \frac{1}{2}
$$

where $\epsilon^{\prime}\left(\frac{1}{2}\right)=-\epsilon^{\prime}\left(-\frac{1}{2}\right)=-1$. A state vector in this $\mathcal{H}_{2}$ is usually called a qubit in quantum information theory [16].

The Hilbert space $\mathcal{H}_{2^{N}}$ is isomorphic to $\left(\mathbb{C}^{2}\right)^{\otimes N}$ and describes a physical system consisting of $N$ qubits, each qubit with two linearly independent states. It has an orthonormal basis denoted by Dirac kets $\left|\Phi_{k}\right\rangle, 1 \leq k \leq 2^{N}$ which are tensor products of $\left| \pm \frac{1}{2}\right\rangle$ by

$$
\left|\Phi_{k}\right\rangle \equiv\left|m_{1}, \cdots, m_{N}\right\rangle \equiv\left|m_{1}\right\rangle \otimes \cdots \otimes\left|m_{N}\right\rangle, \quad m_{1}, \cdots, m_{N}= \pm \frac{1}{2} .
$$

Here the lower index $k$ is a given function of $m_{1}, \cdots m_{N}$, see [30],

$$
k\left[m_{1}, \cdots, m_{N}\right]=2^{N-1}+\frac{1}{2}-\sum_{i=1}^{N} 2^{N-i} m_{i},
$$


so that in coordinates

$$
\left|\Phi_{k}\right\rangle=(\underbrace{0, \cdots, 0}_{k-1}, 1, \underbrace{0, \cdots, 0}_{2^{N}-k})^{T}
$$

where ${ }^{T}$ denotes transpose.

This orthonormal basis $\left|\Phi_{k}\right\rangle$ is partitioned into two sets respectively denoted by Dirac kets $\left|\Phi_{l}\right\rangle$ and $\left|\Phi_{\bar{l}}\right\rangle, 1 \leq l \leq 2^{N-1}$ and $\bar{l}=2^{N}-l+1$,

$$
\left|\Phi_{l}\right\rangle=\left|m_{1}, \cdots, m_{N}\right\rangle, \quad\left|\Phi_{\bar{l}}\right\rangle=\left|\bar{m}_{1}, \cdots, \bar{m}_{N}\right\rangle, \quad \bar{m}_{i}=-m_{i}, 1 \leq l \leq N .
$$

In terms of $\left|\Phi_{l}\right\rangle$ and $\left|\Phi_{\bar{l}}\right\rangle$, the Hilbert space $\mathcal{H}_{2^{N}}$ is spanned by the $2^{N}$ orthonormal GHZ states $\left|\Psi_{l}\right\rangle$ of $N$ qubits,

$$
\left|\Psi_{l}\right\rangle \equiv \frac{1}{\sqrt{2}}\left(\left|\Phi_{l}\right\rangle+\left|\Phi_{\bar{l}}\right\rangle\right), \quad\left|\Psi_{\bar{l}}\right\rangle \equiv \frac{1}{\sqrt{2}}\left(\left|\Phi_{l}\right\rangle-\left|\Phi_{\bar{l}}\right\rangle\right) .
$$

These GHZ states are maximally entangled states that have been widely used in quantum information theory [11, 14, 15. The set of all GHZ states forms an orthonormal basis of $\mathcal{H}_{2^{N}}$.

As an example, consider the Hilbert space $\mathbb{C}^{2} \otimes \mathbb{C}^{2}$ for two qubits (here GHZ states are the well-known Bell states). In terms of the orthonormal product basis $\left|\Phi_{k}\right\rangle, k=1, \cdots, 4$,

$$
\begin{aligned}
& \left|\Phi_{1}\right\rangle=\left|\frac{1}{2}, \frac{1}{2}\right\rangle, \quad\left|\Phi_{4}\right\rangle=\left|\Phi_{\overline{1}}\right\rangle=\left|-\frac{1}{2},-\frac{1}{2}\right\rangle, \\
& \left|\Phi_{2}\right\rangle=\left|\frac{1}{2},-\frac{1}{2}\right\rangle, \quad\left|\Phi_{3}\right\rangle=\left|\Phi_{\overline{2}}\right\rangle=\left|-\frac{1}{2}, \frac{1}{2}\right\rangle,
\end{aligned}
$$

where the numbering for lower indices is consistent with the convention (4.4),

$$
k\left[\frac{1}{2}, \frac{1}{2}\right]=1, k\left[\frac{1}{2},-\frac{1}{2}\right]=2, k\left[-\frac{1}{2}, \frac{1}{2}\right]=3, k\left[-\frac{1}{2},-\frac{1}{2}\right]=4,
$$

Bell states have the same formulations as their conventions,

$$
\begin{aligned}
& \left|\Psi_{1}\right\rangle=\frac{1}{\sqrt{2}}\left(\left|\Phi_{1}\right\rangle+\left|\Phi_{\overline{1}}\right\rangle\right), \quad\left|\Psi_{4}\right\rangle=\left|\Psi_{\overline{1}}\right\rangle=\frac{1}{\sqrt{2}}\left(\left|\Phi_{1}\right\rangle-\left|\Phi_{\overline{1}}\right\rangle\right), \\
& \left|\Psi_{2}\right\rangle=\frac{1}{\sqrt{2}}\left(\left|\Phi_{2}\right\rangle+\left|\Phi_{\overline{2}}\right\rangle\right), \quad\left|\Psi_{3}\right\rangle=\left|\Psi_{\overline{2}}\right\rangle=\frac{1}{\sqrt{2}}\left(\left|\Phi_{2}\right\rangle-\left|\Phi_{\overline{2}}\right\rangle\right) .
\end{aligned}
$$

\subsection{Unitary braid representations for GHZ states}

It is not difficult (mathematically) to construct unitary operators $U$ on $\mathcal{H}_{2^{N}}$ that generate the GHZ states $\left|\Psi_{j}\right\rangle$ from the product basis $\left|\Phi_{k}\right\rangle$. In quantum circuits, the gate operators are physically realized as unitary evolutions of some system. For braiding gates, we particularly want the evolution operator $U$ to satisfy the generalized Yang-Baxter equations (2.11), in order to give rise to representations of the braid group. 


\subsubsection{An even number of qubits}

We recapitulate the results of [8] for GHZ states of an even number $2 n$ of qubits. These are associated to the (generalized) Bell matrix $B^{J J}$ in terms of the almostcomplex structure $M^{J J}$ in Class(1),

$$
B^{J J}=\mathbb{1}_{(2 k)^{2}}+M^{J J}, \quad J=k-\frac{1}{2}, \quad k \in \mathbb{N} .
$$

An important point is that $B^{J J}$ is a $(2 k)^{2} \times(2 k)^{2}$ matrix, while the dimension of the Hilbert space spanned by the GHZ states of $2 n$ qubits is $2^{2 n}$. So for the GHZ states generated by $B^{J J}$ to span a $2^{2 n}$-dimensional Hilbert space, one needs $J=2^{n-1}-\frac{1}{2}$.

Thus for Class (1) with unimodular deformation parameters are chosen to be 1 , which give rise to the unitary braid representation $B_{2^{2 n}}^{J J}$ :

$$
B_{2^{2 n}}^{J J}=\frac{1}{\sqrt{2}}\left(\mathbb{1}_{2^{2 n}}+M_{2^{2 n}}^{J J}\right), \quad M_{2^{2 n}}^{J J}=\sqrt{-1} \sigma_{y} \otimes \sigma_{x}^{\otimes 2 n-1} .
$$

The GHZ states of $2 n$ qubits obtained by the corresponding Bell matrix on the orthonormal product basis $\left|\Phi_{k}\right\rangle$ with $\epsilon^{\prime}\left(m_{1}\right)$ as in (4.2) are

$$
\frac{1}{\sqrt{2}}\left(\left|m_{1}, \cdots, m_{2 n}\right\rangle+\epsilon^{\prime}\left(m_{1}\right)\left|\bar{m}_{1}, \cdots, \bar{m}_{2 n}\right\rangle\right)
$$

which leads to the unitary basis transformation matrix,

$$
B_{2^{2 n}}^{J J}=\left(\left|\Psi_{2^{2 n}}\right\rangle,\left|\Psi_{2^{2 n}-1}\right\rangle, \cdots,\left|\Psi_{2}\right\rangle,\left|\Psi_{1}\right\rangle\right) .
$$

\subsubsection{An odd number of qubits}

Obviously, unitary braid representations in Class (1) can not yield the GHZ states of an odd number, say $2 n+1$, of qubits. But the unitary braid representations in Class (2) can, via the matrices

$$
B_{2^{2 n+1}}=\frac{1}{\sqrt{2}}\left(\mathbb{1}_{2^{2 n+1}}+M_{2^{2 n+1}}\right), \quad M_{2^{2 n+1}}=\sqrt{-} 1 \sigma_{y} \otimes\left(\sigma_{x}\right)^{\otimes 2 n},
$$

The essential differences between unitary braid representations in Class (1) and Class (2) are: every strand for the braid group in Class (1) lives in the same dimensional vector space, whereas this is not always true in Class (2), e.g., for the Bell matrix $B_{2^{2 n+1}}$.

The GHZ states of $2 n+1$ qubits generated by the Bell matrix $B_{2^{2 n+1}}$ in Class (2) acting on the product basis $\left|\Phi_{l}\right\rangle$ and $\left|\Phi_{\bar{l}}\right\rangle$ have a similar form as shown in (4.13), and $B_{2^{2 n+1}}$ represents the unitary basis transformation matrix by

$$
B_{2^{2 n+1}}=\left(\left|\Psi_{2^{2 n+1}}\right\rangle,\left|\Psi_{2^{2 n}}\right\rangle, \cdots,\left|\Psi_{2}\right\rangle,\left|\Psi_{1}\right\rangle\right) .
$$


For example, on the product basis $\left|\Phi_{l}\right\rangle$ for three qubits the generalized Bell matrix obtained from the almost complex structure $M_{8}=\sqrt{-1} \sigma_{y} \otimes \sigma_{x}^{\otimes 2}$ takes the form

$$
B_{8}=\frac{1}{\sqrt{2}}\left(\begin{array}{llllllll}
1 & 0 & 0 & 0 & 0 & 0 & 0 & 1 \\
0 & 1 & 0 & 0 & 0 & 0 & 1 & 0 \\
0 & 0 & 1 & 0 & 0 & 1 & 0 & 0 \\
0 & 0 & 0 & 1 & 1 & 0 & 0 & 0 \\
0 & 0 & 0 & -1 & 1 & 0 & 0 & 0 \\
0 & 0 & -1 & 0 & 0 & 1 & 0 & 0 \\
0 & -1 & 0 & 0 & 0 & 0 & 1 & 0 \\
-1 & 0 & 0 & 0 & 0 & 0 & 0 & 1
\end{array}\right)
$$

which produces all the GHZ states $\left|\Psi_{l}\right\rangle$ of three qubits [11, 14, 15] and satisfies the conditions of Theorem 3.21 with $k=2$. Thus for each $m \geq 1$ one obtains 1) $2^{2 m+1}$-dimensional representations of $\mathcal{B}_{m}$ so that 2) the action of the braid generators on the product basis produces (higher dimensional promotions of) GHZ states on three qubits.

As a basis change operator $B_{8}$ takes the basis $\left|\Phi_{l}\right\rangle$ (with the usual ordering) to

$$
\left(\left|\Psi_{\overline{1}}\right\rangle,\left|\Psi_{\overline{2}}\right\rangle,\left|\Psi_{\overline{3}}\right\rangle,\left|\Psi_{\overline{4}}\right\rangle,\left|\Psi_{4}\right\rangle,\left|\Psi_{3}\right\rangle,\left|\Psi_{2}\right\rangle,\left|\Psi_{1}\right\rangle\right) .
$$

\subsection{Unitary evolution of GHZ states}

Unitary evolution of GHZ states as well as the corresponding Schödinger equation can be explored with the help of Yang-Baxterization [12, 13], and this is a systematic elaboration of previous research work. Unitary evolution of Bell states have been discussed in detail [3, 4, while unitary evolution of GHZ states have been only briefly sketched in 8 .

The quantum Yang-Baxter equation (QYBE) is of the form

$$
\check{R}_{i}(x) \check{R}_{i+1}(x y) \check{R}_{i}(y)=\check{R}_{i+1}(y) \check{R}_{i}(x y) \check{R}_{i+1}(x)
$$

with $x$ or $y$ the spectral parameter. It is well known that one can set up an integrable model by following a given recipe in terms of a solution of the QYBE, see [9, 10]. At $x=y=0$, obviously, the QYBE reduces to $\check{R}_{i} \check{R}_{i+1} \check{R}_{i}=\check{R}_{i+1} \check{R}_{i} \check{R}_{i+1}$, the same as the braid group relation (2.7), $b_{i} b_{i+1} b_{i}=b_{i+1} b_{i} b_{i+1}$. Hence a solution $\check{R}(x)$ of the QYBE always reduces to a braid group representation $b=\check{R}(0)$. In other words, $\check{R}(0)=b$ can be regarded as the asymptotic condition of a solution $\check{R}(x)$ of the QYBE. Conversely, similar to a procedure of solving a differential equation with specified initial-boundary conditions, Baxterization [12] or YangBaxterization [13, represents a procedure of constructing a solution $\check{R}(x)$ of the QYBE (4.19) with the asymptotic condition, $\check{R}(0)=b$, where the braiding $b$ matrix has been specified. For example, for a $b$-matrix with two distinct eigenvalues $\lambda_{1}$ and $\lambda_{2}$, the corresponding $\check{R}(x)$-matrix obtained with Yang-Baxterization is found to be of the form

$$
\check{R}(x)=b+x \lambda_{1} \lambda_{2} b^{-1} .
$$


Please refer to Appendix A of the paper [4 for more details.

The unitary braid operator $B$ derived from the almost-complex structure $M$ (3.11), has two distinct eigenvalues $\zeta$ and $\zeta^{*}$ and satisfies

$$
(B-\zeta \mathbb{1})\left(B-\zeta^{*} \mathbb{1}\right)=0 .
$$

Using Yang-Baxterization, a solution of the QYBE (4.19) with the asymptotic limit $B$, is

$$
\check{R}(x)=B+x B^{-1}=\frac{1}{\sqrt{2}}(1+x) \mathbb{1}+\frac{1}{\sqrt{2}}(1-x) M .
$$

where the lower indices of $B, M, \mathbb{1}$ are suppressed for convenience. This $\check{R}(x)$ matrix can be updated to be a unitary matrix $B(x)$ by adding a normalization factor $\rho(x)$, i.e.,

$$
B(x)=\rho^{-\frac{1}{2}} \check{R}(x), \quad \rho=1+x^{2}, x \in \mathbb{R} .
$$

As the real spectral parameter $x$ plays the role of the time variable, the Schrödinger equation describing the unitary evolution of a state $\psi(0)$ (independent of $x$ ) determined by the $B(x)$ matrix, i.e., $\psi(x)=B(x) \psi(0)$, has the form

$$
\sqrt{-1} \frac{\partial}{\partial x} \psi(x)=H(x) \psi(x), \quad H(x) \equiv \sqrt{-1} \frac{\partial B(x)}{\partial x} B^{-1}(x),
$$

where the time-dependent Hamiltonian $H(x)$ is given by

$$
H(x)=\sqrt{-1} \frac{\partial}{\partial x}\left(\rho^{-\frac{1}{2}} \check{R}(x)\right)\left(\rho^{-\frac{1}{2}} \check{R}(x)\right)^{-1}=-\sqrt{-1} \rho^{-1} M .
$$

To construct the time-independent Hamiltonian, the spectral parameter $x$ is replaced by a new time variable $\theta$ by the change of variables

$$
\cos \theta=\frac{1}{\sqrt{1+x^{2}}}, \quad \sin \theta=\frac{x}{\sqrt{1+x^{2}}},
$$

so that the unitary matrix $B(x)$ has a new formulation in terms of $\theta$,

$$
B(\theta)=\cos \theta B+\sin \theta B^{-1}=e^{\left(\frac{\pi}{4}-\theta\right) M},
$$

and hence the Schrödinger equation for the time evolution of $\psi(\theta)=B(\theta) \psi(0)$ is given by

$$
\sqrt{-1} \frac{\partial}{\partial \theta} \psi(\theta)=H \psi(\theta), \quad H \equiv \sqrt{-1} \frac{\partial B(\theta)}{\partial \theta} B^{-1}(\theta)=-\sqrt{-1} M,
$$

where the time-independent Hamiltonian $H$ is Hermitian since the almost-complex structure $M$ is anti-Hermitian. The unitary time-evolution operator $U(\theta)$ has the form $U(\theta)=e^{-\theta M}$. Furthermore, with the shifted time variable $\theta^{\prime}$, unitary matrices $B\left(\theta^{\prime}\right)$ and $U\left(\theta^{\prime}\right)$ take the same form,

$$
B\left(\theta^{\prime}\right)=U\left(\theta^{\prime}\right)=e^{-\theta^{\prime} M}, \quad \theta^{\prime}=\theta-\frac{\pi}{4} .
$$


In Class (1), Yang-Baxterization of the Bell matrix $B^{J J}$ for GHZ states of $2 n$ qubits has the form

$$
B^{J J}\left(\theta^{\prime}\right)=\cos \theta^{\prime} \mathbb{1}_{2^{2 n}}-\sin \theta^{\prime} M_{2^{n}} \otimes P_{2^{n}} .
$$

Define $|\alpha\rangle$ by

$$
|\mu\rangle:=\left|\Phi_{\mu}\right\rangle, \quad|\nu\rangle:=\left|\Phi_{\nu}\right\rangle, \quad|\alpha\rangle:=|\mu \nu\rangle=\left|\Phi_{(\mu-1) 2^{n}+\nu}\right\rangle .
$$

Then the unitary evolution of the GHZ state $|\alpha\rangle$ is given by

$$
B^{J J}\left(\theta^{\prime}\right)|\alpha\rangle=\cos \theta^{\prime}|\alpha\rangle-\sin \theta^{\prime} \epsilon(\mu) e^{\frac{\varphi_{\mu}+\varphi_{v}}{2}}|\bar{\alpha}\rangle
$$

corresponding to the Hamiltonian $H^{J J}=-\sqrt{-1} M_{2^{n}} \otimes P_{2^{n}}$.

In Class (2), Yang-Baxterization of the Bell matrix $B_{2^{n}}$ has the form in terms of the Hamiltonian $H_{2^{n}}$,

$$
B_{2^{n}}\left(\theta^{\prime}\right)=e^{-\sqrt{-1} \theta^{\prime} H_{2^{n}}}, \quad H_{2^{n}}=\sigma_{y} \otimes \sigma_{x}^{\otimes n-1}
$$

which derives the unitary evolution of GHZ state $\left|\Psi_{l}\right\rangle$ defined by (4.7),

$$
B_{2^{n}}\left(\theta^{\prime}\right)\left|\Phi_{l}\right\rangle=\cos \theta^{\prime}\left|\Phi_{l}\right\rangle-\sin \theta^{\prime} \epsilon^{\prime}\left(m_{1}\right)\left|\Phi_{\bar{l}}\right\rangle .
$$

For example, the unitary evolution of GHZ states of three qubits determined by $B_{8}\left(\theta^{\prime}\right)=e^{-\sqrt{-1} \theta^{\prime} H_{8}}$ have the following realization,

$$
B_{8}\left(\theta^{\prime}\right)\left|\frac{1}{2} \frac{1}{2} \frac{1}{2}\right\rangle=\cos \theta^{\prime}\left|\frac{1}{2} \frac{1}{2} \frac{1}{2}\right\rangle+\sin \theta^{\prime}\left|\frac{-1}{2} \frac{-1}{2} \frac{-1}{2}\right\rangle, \quad H_{8}=\sigma_{y} \otimes \sigma_{x}^{\otimes 2} .
$$

\section{Conclusions and Discussions}

In this paper we have revealed the connections between a special class of multiqubit braiding quantum gates and extraspecial 2-groups. More concretely, we have shown that one may associate to certain almost-complex structures antiHermitian representations of extraspecial 2-groups, which in turn give rise to unitary representations of the braid group factoring over extensions of 2-groups by symmetric groups. These unitary braid representations can be used to generate the maximally entangled GHZ states for an arbitrary number of qubits. Since the braiding quantum gates are known to play a pivotal role in topological quantum computation 2, 18, 19, 21, our present work suggests that extraspecial 2-groups should play an important role in topological quantum computation, at least in the analysis of quantum circuits consisting of the braiding gates that we have studied.

For example, in the Freedman-Kitaev topological model [18, 19] for quantum computation, the gates are realized as operators representing braids in $2+1$ dimensions. Given a gate $U$ it is a difficult problem to find a braid that (even approximately) realizes $U$. Indeed, the ubiquitous entangling CNOT gate is quite 
difficult to achieve in this setting (see e.g. [31]). It is not known if the CNOT gate can be exactly realized in some model. Our results by exploring extraspecial 2-groups suggest that in the $S U(2)_{2}$ model for the topological state (see [24]) realized as the state at filling fraction $\nu=5 / 2$ in the fractional quantum Hall effect, one may obtain all GHZ states exactly. In this sense the generalized Bell matrices we have studied here are as important to topological quantum computation as the CNOT gate is to the quantum circuit model.

We also expect that extraspecial 2-groups play an important role in the theory of quantum error correction, which protects quantum information against noises. On one hand, the extraspecial 2-groups provide a bridge between quantum error correcting codes and binary orthogonal geometry [32. On the other hand, they form a subgroup of the Pauli group [33, which plays a crucial role in the theory of stabilizer codes 34. Therefore, the new connection, that we have revealed in this paper, between extraspecial 2-groups and braid group representations suggests possible applications of the multi-qubit unitary braiding quantum gates in quantum error correction codes. In particular, the Jones braid representations at a 4-th root of unity is known to be closely related to the representations of extraspecial 2-groups [5]. While the finiteness of the braid group image precludes the associated braiding gates alone from forming a universal gate set (in the sense of [35]), this new connection suggests in turn that quantum systems with braiding statistics modeled by the Jones representation at 4-th roots of unity may be used for quantum error correction.

Finally we conclude with a mathematical remark. Goldschmidt and Jones [36] use extraspecial $p$-groups (Heisenberg groups) to construct braid group (specialized Burau-Squier) representations factoring over finite symplectic groups. Although they work exclusively over fields of odd characteristic and hence only extraspecial $p$-groups with $p \neq 2$ appear, our work may be thought of as a characteristic-2 complement to [36] in some respects, particularly as link invariants are concerned.

\section{Acknowledgments}

We thank Zhenghan Wang for participating in the early stage of this work. Y. Zhang thanks L.H. Kauffman for stimulating discussions and S.L. Braunstein for helpful comments. E. Rowell was supported in part by NSA grant H98230-08-10020. Y. Zhang was supported in part by the Seed Grant of University of Utah and NSFC-10605035. Y.S. Wu was supported in part by U.S. NSF through Grant No. PHY-0407187.

Note Added: Deformations with phase parameters similar to those appearing in eq. (3.12) have been used in the context of deformed exchange statistics in 1D systems, leading to a deformed solution of the Yang-Baxter equation [37. Also the matrix (3.11) recently appears in the context of an SL(2) invariant extension of the entanglement measure concurrence to higher (half-integer) spins in ArXiv. 
0908.3818 ([38]). We thank the referee for pointing out these references to us.

\section{A The generalized quantum Yang-Baxter equation}

The $\check{R}_{i}(x)$-matrix in terms of the representation $\phi_{n-1}\left(e_{i}\right)$ of the extraspecial 2group $\mathbf{E}_{n}$ of the form

$$
\check{R}_{i}(x)=\frac{1}{\sqrt{2}}\left((1+x) \mathbb{1}+(1-x) \phi_{n-1}\left(e_{i}\right)\right)
$$

can be shown to satisfy the QYBE (4.19). The proof is by calculation:

$$
\begin{aligned}
& \sqrt{2} \check{R}_{i}(x) \check{R}_{i+1}(x y) \check{R}_{i}(y)=((1+x y)(x+y) \mathbb{1} \\
& +(1-x y)(y-x) \phi_{n-1}\left(e_{i}\right) \phi_{n-1}\left(e_{i+1}\right) \\
& \left.+(1+x y)(1-x y)\left(\phi_{n-1}\left(e_{i}\right)+\phi_{n-1}\left(e_{i+1}\right)\right)\right)
\end{aligned}
$$

which is symmetric under $i \leftrightarrow i+1$ and $x \leftrightarrow y$ so that $\check{R}_{i}(x)$ satisfies (4.19).

In the Class (1) representation for $\mathbf{E}_{n}$, equation (4.19) is the standard version of the QYBE exploited in the literature, i.e.,

$\left(\check{R}(x) \otimes \mathbb{1}_{2 k}\right)\left(\mathbb{1}_{2 k} \otimes \check{R}(x y)\right)\left(\check{R}(y) \otimes \mathbb{1}_{2 k}\right)=\left(\mathbb{1}_{2 k} \otimes \check{R}(y)\right)\left(\check{R}(x y) \otimes \mathbb{1}_{2 k}\right)\left(\mathbb{1}_{2 k} \otimes \check{R}(x)\right)$

where $\check{R}_{i}(x)$ is an invertible $2 k \otimes 2 k$ matrix. However in the Class (2) representation for $\mathbf{E}_{n}$, equation (4.19) is an example of a generalized version of the QYBE given by

$$
\left(\check{R}(x) \otimes \mathbb{1}_{l}\right)\left(\mathbb{1}_{l} \otimes \check{R}(x y)\right)\left(\check{R}(y) \otimes \mathbb{1}_{l}\right)=\left(\mathbb{1}_{l} \otimes \check{R}(y)\right)\left(\check{R}(x y) \otimes \mathbb{1}_{l}\right)\left(\mathbb{1}_{l} \otimes \check{R}(x)\right)
$$

where $l=p^{k}(2 \leq p \in \mathbb{N})$ and $\check{R}(x)$ is an invertible $p^{N} \otimes p^{N}$ matrix, in other words, (4.19) is a Yang-Baxterized version of the generalized YBE (2.11).

By reparameterization, the above $\check{R}(x)$-matrix has an alternative expression, $\check{R}_{i}(\Theta)$, given by

$$
\check{R}_{i}(\Theta)=\mathbb{1}+\tanh \Theta \phi_{n-1}\left(e_{i}\right)
$$

satisfying the parameter-additive (instead of multiplicative) form of the QYBE (4.19),

$$
\check{R}_{i}\left(\Theta_{1}\right) \check{R}_{i+1}\left(\Theta_{1}+\Theta_{2}\right) \check{R}_{i}\left(\Theta_{2}\right)=\check{R}_{i+1}\left(\Theta_{2}\right) \check{R}_{i}\left(\Theta_{1}+\Theta_{2}\right) \check{R}_{i+1}\left(\Theta_{1}\right) .
$$

This form is more closely related to elastic or inelastic scattering phenomena in quantum physics. Interested readers are invited to refer to [7, 8].

\section{References}

[1] H.A. Dye, Unitary Solutions to the Yang-Baxter Equation in Dimension Four, Quant. Inf. Proc. 2 (2003) 117-150. Arxiv: quant-ph/0211050. 
[2] L.H. Kauffman and S.J. Lomonaco Jr., Braiding Operators are Universal Quantum Gates, New J. Phys. 6 (2004) 134. Arxiv: quant-ph/0401090.

[3] Y. Zhang, L.H. Kauffman and M.L. Ge, Universal Quantum Gate, YangBaxterization and Hamiltonian. Int. J. Quant. Inform. 3 no. 4 (2005) 669-678. Arxiv: quant-ph/0412095.

[4] Y. Zhang, L.H. Kauffman and M.L. Ge, Yang-Baxterizations, Universal Quantum Gates and Hamiltonians. Quant. Inf. Proc. 4 (2005) 159-197. Arxiv: quant-ph/0502015.

[5] J. Franko, E.C. Rowell and Z. Wang, Extraspecial 2-Groups and Images of Braid Group Representations. J. Knot Theory Ramifications, 15 (2006) 413428. Arxiv: math.RT/0503435.

[6] Y. Zhang, L.H. Kauffman and R.F. Werner, Permutation and its Partial Transpose. Int. J. Quant. Inform. 5 no. 4 (2006) 469-507. Arxiv: quant-ph/0606005.

[7] Y. Zhang, N. Jing and M.L. Ge, Quantum Algebras Associated With Bell States. J. Phys. A: Math. Theor. 41 (2008) 055310; Arxiv: math-ph/0610036.

[8] Y. Zhang and M.L. Ge, GHZ States, Almost-Complex Structure and Yang-Baxter Equation. Quant. Inf. Proc. 6 (2007) 363-379; Arxiv: quant-ph/0701244.

[9] C.N. Yang, Some Exact Results for the Many Body Problems in One Dimension with Repulsive Delta Function Interaction, Phys. Rev. Lett. 19 (1967) 1312-1314.

[10] R.J. Baxter, Partition Function of the Eight-Vertex Lattice Model, Annals Phys. 70 (1972) 193-228.

[11] D.M. Greenberger, M.A. Horne and A. Zeilinger, Going beyond Bell's Theorem, in Bell's Theorem, Quantum Theory, and Conceptions of the Universe, edited by M. Kafatos, pp. 73-76, (Kluwer Academic, Dordrecht, 1989).

[12] V.F.R. Jones, Baxterization, Int. J. Mod. Phys. A6 (1991) 2035-2043.

[13] M.L. Ge, K. Xue and Y-S. Wu, Explicit Trigonometric Yang-Baxterization, Int. J. Mod. Phys. A6 (1991) 3735.

[14] D.M. Greenberger, M.A. Horne, A. Shimony, and A.Zeilinger, Bell's Theorem Without Inequalities, Am. J. Phys. 58 (1990) 1131-1143.

[15] D. Bouwmeester, J.-W. Pan, M. Daniell, H. Weinfurter and A. Zeilinger, Observation of Three-Photon Greenberger-Horne-Zeilinger Entanglement, Phys. Rev. Lett. 82(1999) 1345-1349. 
[16] M. Nielsen and I. Chuang, Quantum Computation and Quantum Information (Cambridge University Press, 1999).

[17] D. Aharonov and M. Ben-Or, Fault tolerant computation with constant error, in Proceedings of the Twenty-Ninth Annual ACM Symposium on the Theory of Computing, pp. 176-188, 1997.

[18] A. Yu. Kitaev, Fault-tolerant Quantum Computation By Anyons, Ann. Phys. 303 (2003) 2. Arxiv: quant-ph/9712048.

[19] M. Freedman, A. Kitaev, M. Larsen and Z. Wang, Topological Quantum Computation, Bull. Amer. Math. Soc. (N.S.) 40 no. 1 (2003) 31-38.

[20] L.H. Kauffman, Knots and Physics (World Scientific Publishers, 2002).

[21] L.H. Kauffman and S.J. Lomonaco Jr, Q-Deformed Spin Networks, Knot Polynomials and Anyonic Topological Computation, J. Knot Theory Ramifications, 16 (2007) 267-332. Arxiv: quant-ph/0606114.

[22] V. F. R. Jones, Braid groups, Hecke algebras and Type $\mathrm{II}_{1}$ Factors, Geometric methods in operator algebras (Kyoto, 1983), 242-273, Pitman Res. Notes Math. Ser., 123, Longman Sci. Tech., Harlow, 1986.

[23] V. F. R. Jones, Hecke Algebra Representations of Braid Groups and Link Polynomials, Ann. Math. 126 (1987), 335-388.

[24] S. Das Sarma, M. Freedman, C. Nayak, S. Simon and A. Stern, NonAbelian Anyons and Topological Quantum Computation, preprint, ArXiv: cond-mat.str-el/0707.1889.

[25] R. Griess, Automorphisms of Extra Special Groups and Nonvanishing Degree Two Cohomology, Pacific J. Math. 48 no. 2 (1973) 403-422.

[26] J.L. Chen, Kang Xue and Mo-Lin Ge, Berry Phase and Quantum Criticality in Yang-Baxter Systems, Ann. of Phys. 323 (2008) 2614.

[27] P.K. Aravind, Borromean Entanglement of the GHZ state, in Potentiality, Entanglement and Passion-at-a-Distance, Cohen, Robert S., Michael Horne, and John Stachel (eds.), Kluwer Academic Publishers, Boston 1997.

[28] B. Abdesselam, A. Chakrabarti, V.K. Dobrev and S.G. Mihov, Higher Dimensional Unitary Braid Matrices: Construction, Associated Structures and Entanglements, J. Math. Phys. 48 (2007) 053508; ArXiv:math/0702188.

[29] J.L. Brylinski and R. Brylinski, Universal Quantum Gates, in Mathematics of Quantum Computation, Chapman \& Hall/CRC Press, Boca Raton, Florida, 2002 (edited by R. Brylinski and G. Chen). 
[30] K. Fujii, H. Oike and T. Suzuki, More on the Isomorphism $S U(2) \otimes S U(2) \cong$ $S O(4)$, Int. J. Geom. Methods Mod. Phys. 4 no. 3 (2007) 471-485.

[31] N. Bonesteel, L Hormozi, G. Zikos, S. Simon, Braid Topologies for Quantum Computation. Phys. Rev. Lett. 95 (2005), no. 14, 140503, 4 pp.

[32] A. Calderbank, E. Rains, P. Shor and N. Sloane, Quantum Error Correction and Orthogonal Geometry, Phys. Rev. Lett. 78 (1997) 405.

[33] A. Calderbank, E. Rains, P. Shor and N. Sloane, Quantum Error Correction via Codes over GF(4), IEEE Transactions on Information Theory, 44(4) (1998) 1369.

[34] D. Gottesman, Stablizer Codes and Quantum Error Correction, Ph.D. Thesis, Caltech, 1997.

[35] M. Freedman, M. Larsen, Z. Wang, The Two-Eigenvalue Problem and Density of Jones Representation of Braid Groups, Comm. Math. Phys. 228 (2002) 177-199. ArXiv: math.GT/0103200.

[36] D.M. Goldschmidt and V.F.R. Jones, Metaplectic Link Invariants, Geom. Dedicata 31 (1989) 165-191.

[37] A. Osterloch, L. Amico, U. Eckern, Bethe Ansatz Solution of a New Class of Hubbard-type Models. J. Phys. A33 (2000) L87; Fermionic Long-range Correlations Realized by Particles Obeying Deformed Statistics. J. Phys. A33 (2000) L487; Exact Solution of Generalized Schulz-Shastry type Models. Nucl. Phys. B 588 (2000) 531.

[38] A. Osterloh, J. Siewert, The Invariant-Comb Approach and Its Relation to the Balancedness of Multipartite Entangled States. ArXiv: 0908.3818. 